\title{
Contributions of muon spin rotation/relaxation/resonance to the understanding of problems in magnetism - Current and future
}

\author{
K NAGAMINE \\ Meson Science Laboratory, Institute of Materials Structure Science, High Energy Accelerator Research Organization \\ (KEK-MSL), Oho, Tsukuba, Ibaraki 305-0801, Japan and \\ Muon Science Laboratory, Institute of Physical and Chemical Research (RIKEN), Wako, Saitama 351-0198, Japan
}

\begin{abstract}
During these 25 years following the start of muon spin rotation/relaxation/resonance ( $\mu$ SR) studies on condensed matter, various new aspects of magnetic materials have been explored by using high sensitivity to the microscopic dynamical behaviour of the host magnetism, specifically, the studies on magnetic ordering in strongly-correlated electron systems, elementary excitations in conducting polymers and protein, etc. Intense (more than $10^{6} / \mathrm{cm} \cdot \mathrm{s}$ ) and ultra-slow (below $\mathrm{keV}$ ) muon beams to be available at future accelerator facilities may open new areas such as spin dynamics studies of surface magnetism, cluster particles, etc.
\end{abstract}

Keywords. Muon spin rotation/relaxation/resonance; magnetism.

\section{Introduction}

The principle of the muon spin rotation/relaxation/resonance $(\mu \mathrm{SR})$ method is based upon the laws of particle physics. Figure 1 shows the spin of the $\mu^{+}$(positive muon), when it is born by decay of the $\pi^{+}$(positive pion), which is completely polarized along the direction of its motion; once the $\mu^{+}$is obtained as a beam, it is polarized along the beam direction. During a slowingdown of the $\mu^{+}$inside the host material, the spin polarization is maintained. After thermalization and occupation at the specific microscopic location, the $\mu^{+}$ decays into $e^{+}$and two neutrinos. The $e^{+}$takes a spatial distribution preferentially along the $\mu^{+}$spin; and the $e^{+}$energy goes up to $50 \mathrm{MeV}$. This helps to detect the direction of the $\mu^{+}$spin time by time by using $e^{+}$detectors placed outside the target material to be investigated under various condensed-matter experimental environments e.g. low- or high-temperature, high pressure, etc.

A schematic representation of the actual experimental arrangement is given in figure 2. A sample, with a typical size of a few $\mathrm{cm}^{2}$ area and a few $100 \mathrm{mg} / \mathrm{cm}^{2}$ thickness, is placed along the $\mu^{+}$beam direction. The sample is surrounded by an array of the $e^{+}$detections and in the case of $\mu \mathrm{SR}$ experiments with intense pulsed muon beam, the $e^{+}$detector is highly segmented to eliminate a counting loss. A time-digitizer and an on-line data taking system are connected to measure timedependence of the anisotropic $e^{+}$intensity with reference to the time of the $\mu^{+}$arrival; one can take time-dependence of the intensity ratio between forward and backward counters to measure the $e^{+}$asymmetry to monitor a time evolution of the $\mu^{+}$spin polarization.
Based on the theoretical developments initiated by Prof. Kubo, the observed time evolution of the $\mu^{+}$spin direction, seen in a time spectrum of the asymmetry of anisotropic $e^{+}$decay, can have an one-to-one correspondence with the static as well as the dynamic nature of the microscopic magnetic field seen by the $\mu^{+}$(see figure 2).

Thus, the $\mu$ SR method can be considered as a sensitive magnetic compass to probe microscopic magnetic properties of the condensed matter. The excellent features of the $\mu \mathrm{SR}$ method, compared to the other microscopic probes like neutron scattering, synchrotron radiation, NMR, etc. can be summarized as follows: (i) because of spin polarization, which is based on particlephysics laws, microscopic magnetic properties can be studied under zero external field, a significant advantage for the studies of superconductors, (ii) sensitive measurements of the microscopic fields with a capability of a high efficiency detection of radioactive product can be realized by employing high flux of the $\mu^{+}$ beam wherein, e.g., $1 \mathrm{M} e^{+}$events/min can be obtained, (iii) with the help of the large $\mu^{+}$magnetic moment (3.2 times that of the proton) as well as a 'short' life time nature of the $\mu^{+}(2.2 \mu \mathrm{s})$, the $\mu$ SR method is sensitive to the very weak (down to less than a Gauss) and a randomly oriented microscopic magnetic field and (iv) because of the large magnetic moment as well as the available time-window for the $\mu \mathrm{SR}$ measurement, the $\mu S R$ is sensitive to the dynamics of the surrounding electronic spin whose characteristic correlation time is somewhat slower than that detected by neutron scattering and faster than that detected by NMR. 


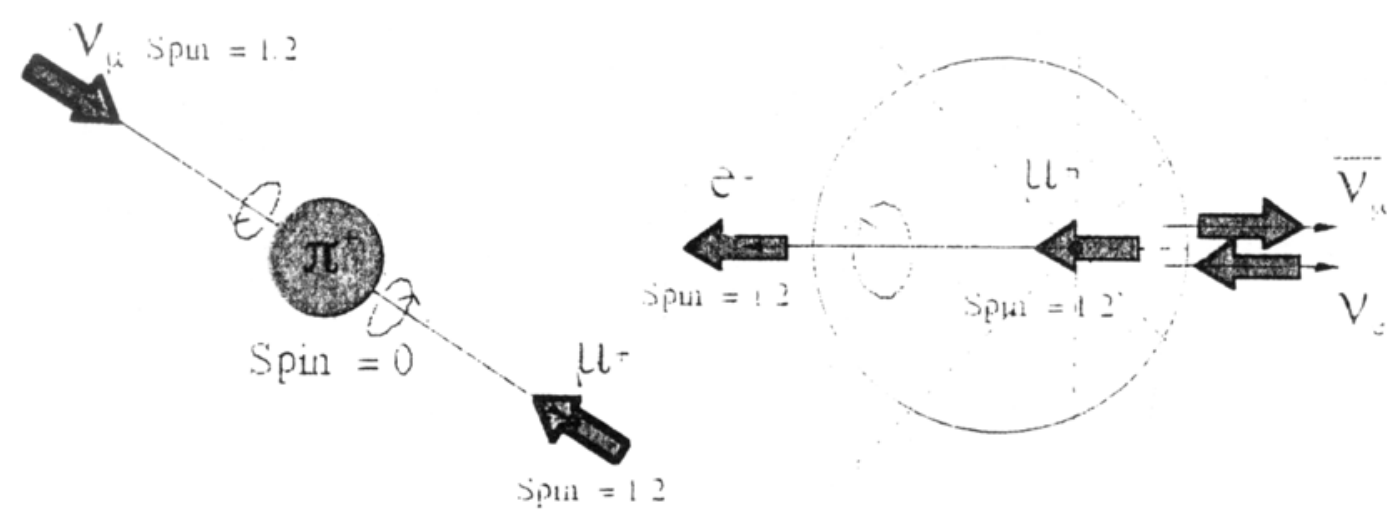

Figure 1. Muon spin polarization originating from positive pion decay (left). Spatial distribution of positron from the decay of polarizated muon (right).

\section{Current active topics}

The $\mu$ SR method has been applied to the various condensed matter studies as listed in table 1 . As given in the list, there are two types of experimental studies: (a) probing the original microscopic magnetic structure in the target material by taking the $\mu^{+}$as a probe with minimum or zero perturbations: and (b) considering the $\mu^{+}$as a creator of the new microscopic order by an introduction of a positively charged light mass.

There have been significant progresses in the $\mu S R$ applications to the understandings of magnetism and magnetic materials in these years. Up to 1996 , details of these progresses can be found in the proceedings of the latest international conference on the $\mu \mathrm{SR}$ (Nagamine 1997).

In this paper we have highlighted the researches after 1996. We have described rather restricted topics, namely, (i) magnetic ordering in strongly correlated electron systems, (ii) spin dynamic in conducting polymers, and (iii) rapid electron transfer in protein.

\subsection{Magnetic orderings in strongly correlated electron systems}

Historically, the usefulness of the $\mu$ SR method in condensed matter studies was acknowledged in late 80 s by a series of measurements of the magnetic phase diagramme of high $T_{\mathrm{C}}$ superconductor (Nishida et at 1987) as well as by the observation that there is ordering of small magnetic moments in heavy Fermion systems (Amato 1997). Both of these studies have been extended under the name of $\mu S R$ studies on strongly correlated electron systems. In addition, after 1995, the colossal magneto-resistance materials have also been included in these studies. In this section we will summarize typical examples of these studies mainly from our viewpoint.

First, we describe how the basic microscopic magnetic properties can be studied by the $\mu$ SR method, by taking $\mu^{+} \mathrm{SR}$ studies on high $T_{\mathrm{C}} \mathrm{LaSrCuO}$ as an example (Torikai et al 1993).

For the $\mu^{+}$site determination, the location of the $\mu^{+}$ should be determined without using the host magnetic properties. For this end, the only consistent method is to use $\mu^{+}$spin relaxation which results due to the surrounding nuclear magnetic moments in the paramagnetic phase where fluctuating electronic moments do not contribute at least significantly to the $\mu^{+}$relaxation. By using a single crystal of $\mathrm{LaSrCuO}$, the $\mu^{+}$location was thus determined to be $1 \mathrm{~A}$ below the apical oxygen.

The $\mu^{+}$hyperfine field and nature of electron spin system can be studied by taking the well-understood system where the $\mu^{+}$local field should be studied by measuring the field vector (strength and direction) in order to learn hyperfine interactions between the localized $\mu^{+}$and the surrounding electronic spin. By using antiferro $\mathrm{La}_{2} \mathrm{CuO}_{4}$, the $\mu^{+}$field vector as well as the spin of the $\mathrm{Cu}$ moment have been determined.

Studies of magnetic phase 'diagramme including the hole (electron)-doping dependence can be studied by using various samples where the $\mu^{+}$location can be assumed to remain almost unchanged and the $\mu^{+}$hyperfine field changes systematically from the undoped system. The occurrence of magnetic order, either static or dynamic, can be studied. The hole dependence of magnetic properties has been studied in $\mathrm{La}_{1-x} \mathrm{Sr}_{x} \mathrm{CuO}_{4}$ as a function of hole concentration $(x)$.

2.1a The 1/8 problem in high $T_{C}$ superconductor: The so-called $1 / 8$ problem in high $T_{\mathrm{C}}$ superconductor has been investigated according to the following steps.

In $\mathrm{LaBa}$, for $0.05<x<0.28$ where the system is superconductor, the $T_{\mathrm{c}}$ suppression was discovered at $x=0.125(1 / 8)$, where the crystal structure change from LTO to LTT occurs at the temperature above $T_{\mathrm{c}}$. The experiment using the $\mu$ SR (Kumagai et al 1991) and later using NMR and neutrons showed an antiferromagnetic order at $x=0.125$. 
In $\mathrm{LaSr}$, by replacing $40 \%$ of $\mathrm{La}$ to $\mathrm{Nd}$, at $x=0.125$ and below the LTO to LTT structure change with a $T_{\mathrm{C}}$ suppression, neutron scattering has shown antiferromagnetic ordering as well as a stripe structure of charge and spin (Tranquada et al 1995).

In the original $\mathrm{LaSr}$ without any replacements of $\mathrm{La}$, which does not have any crystal structure change, a spin-glass order has been suggested without $T_{\mathrm{C}}$ suppre-

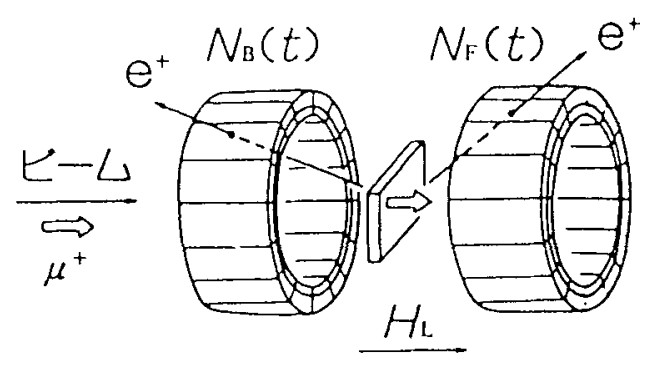

TIME EVOLUTION OF MUON DECAY ASYMMETRY A(t)

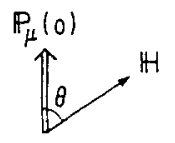

$$
\sigma_{Z}(t)=\cos ^{2} \theta+\sin ^{2} \theta \cos Y_{\mu} H t
$$

$A(t)=\int \sigma_{Z}(t) P\left(H_{x}\right) P\left(H_{y}\right) P\left(H_{z}\right) d H_{x} d H_{y d} H_{z}$

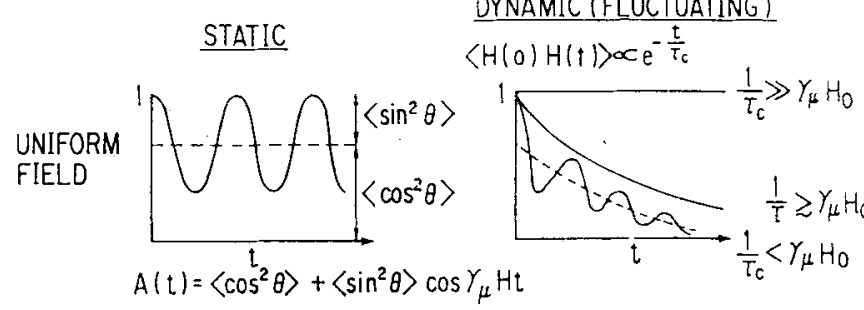
DYNAMIC (FLUCTUATING)
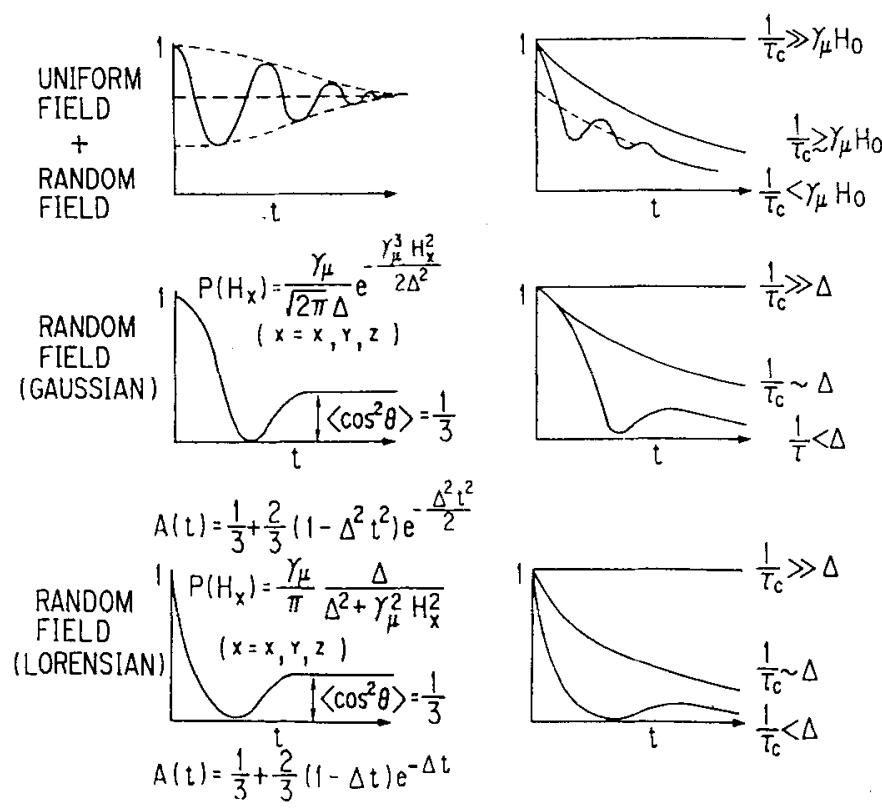

Figure 2. Typical layout of $\mu$ SR experiment with pulsed muons (above). The $\mu$ SR time spectrum expected from the magnitude, distribution and fluctuation of the local field at the muon (below). ssion at $x=0.115$ (Torikai 1993), while antiferromagnetic ordering has been observed with $T_{\mathrm{c}}$ suppression at $x=0.115$ (Watanabe et al 1994). Also, in recent neutron, NMR, supersonic measurements at $x=0 \cdot 12$, existences of magnetic superlattice, magnetic order in La nuclei as well as softening of longitudinal sonic wave have been detected, suggesting a dynamical pinning of spin correlations by a lattice instability towards LTT (Suzaki et al 1998).

Recently the anomaly in the original LaSr at $x=0.115$ has seriously been revisited by comparing the existing two sets of data on the two samples to the data obtained for the new and refined sample made by the Tokyo University of Science group (Arai 1998). We can summarize these observations as: (i) there is antiferromagnetic ordering at $x=0.115$ with transition temperature of $10 \mathrm{~K}$, no matter how the system subjects to superconductivity or nonsuperconductivity; and (ii) the occurrence of antiferromagnetic ordering happens almost commonly in all these three samples (figure 3 ).

All these suggest that at $x=0 \cdot 115$, instead of $x=0.125$, the original LaSr may take an unique antiferromagnetic ordering whose origin is totally an open problem.

The 1/8 problem in addition to the La-based high $T_{\mathrm{C}}$ oxides, appears to exist in $\mathrm{BiSrCuO}$ system as well where an occurrence of the dynamical relaxation of the $\mu^{+}$spin was seen at $0.3 \mathrm{~K}$ for the $\mathrm{Ca}$ and $\mathrm{Zn}$ doping sample with effective hole doping of $x=0.125$. The presently known $1 / 8$ problem is summarized in table 2 .

2.1b Magnetic orderings and fluctuations in heavy fermion systems: Following the progress of sample preparation methods of the $\mathrm{U}$ - as well as Ce-based heavy fermion systems, new and reliable data are now coming out in various types of measurements, including $\mu \mathrm{SR}$ experiments.

Significant example is magnetic ordering in $\mathrm{UPt}_{3}$ at very low temperatures. According to the recent measurements at KEK-MSL by the KEK-RIKEN-TIT-JAERI-

Table 1. List of representative $\mu S R$ condensed matter studies, achieved so far (Spring 1998).

1. Hyperfine structures of interstitial $\mu^{+}$and bound $\mu^{-}$just beside nuclei in ferromagnetic metals

2. Critical phenomena and magnetic ordering in heavy fermion systems and ferro-magnetic metals

3. Spin dynamic in random magnets like spin glass and lowdimension magnets

4. Magnetic phase diagramme, penetration depth, vortex structure in high $T_{\mathrm{C}}$ superconductors

5. Spin dynamic in conducting polymers and macromolecules

6. $\mu^{+}$quantum diffusion in metals and insulators

7. $\mu^{+} /$muonium center and its reaction in semiconductor

8. Ultra-slow $\mu^{+}$generation and its application to surface studies 
OSAKA collaboration, some questions have been raised for the proposed antiferromagnetic ordering below $50 \mathrm{mK}$ as well as for the occurrence of spontaneous magnetism below $T_{\mathrm{c}}$ of $0.53 \mathrm{~K}$ (Higemoto et al 1998).

On the other hand, by using sensitive-probe-nature of spin dynamics, some of the recent $\mu \mathrm{SR}$ experiments have revealed the scaling of $\mu^{+}$spin relaxation corresponding to the non-Fermi liquid ordering in (i) $\mathrm{CeCoGe}_{1-x} \mathrm{Si}_{x}$ $(1.1<x<1.5)$, unique scaling behaviour has been found at KEK and RIKEN-RAL by RIKEN-KEK-UT/ISSP collaboration (Krishnamurthy et al 1999a); and (ii) $\mathrm{Ce}$ $\left(\mathrm{Ru}_{1-x} \mathrm{Rh}_{x}\right)_{2} \mathrm{Si}_{2}(x=0.5)$, the unique scaling with levellingoff behaviour was observed at RIKEN-RAL by the OSAKA-RIKEN-KEK collaboration, suggesting dynamical fluctuations of finite size magnets of tiny moments (Yamamoto et al 1998).

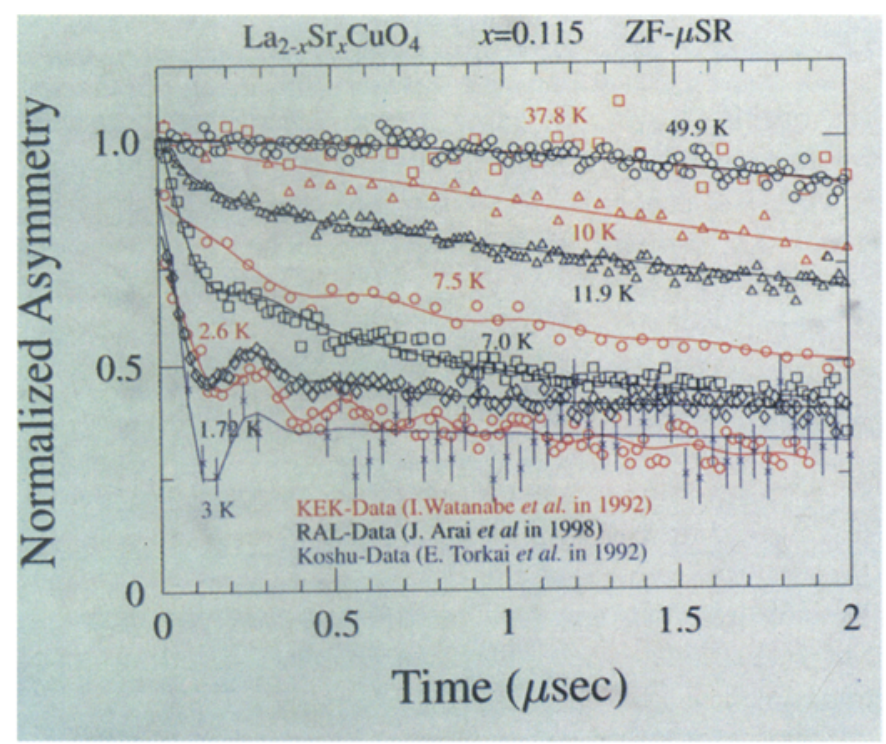

Figure 3. The $\mu \mathrm{SR}$ pattern observed for 3 samples of $\mathrm{LaSrCuO}$ with $x=0 \cdot 115$. 2.1c Magnetic orderings and fluctuations in colossal magneto-resistance materials: The MnO-based distorted perovskite crystals are now attracting attention due to their properties of colossal magneto-resistance. By using $\mu S R$ 's sensitive-probe-nature to the spin dynamics, recent $\mu \mathrm{SR}$ studies at RIKEN-RAL by RIKEN-KEK-JRCAT collaboration have observed a characteristic slowing down behaviour towards the ordering temperature in paramagnetic phase (Krishnamurthy et al 1999b); the $\mathrm{Nd}_{0.5} \mathrm{Sr}_{0.5} \mathrm{MnO}_{3}$ with a CMR behaviour was found to take a characteristic critical slowing-down of $\mathrm{Mn}$ spin fluctuation with some crossover behaviour, while the $\mathrm{Nd}_{0.45} \mathrm{Sr}_{0.55} \mathrm{MnO}_{3}$ without a CMR behaviour was found to take none of such critical behaviours.

\subsection{Spin dynamics in conducting polymers}

The spin dynamics of paramagnetic conduction electrons in conducting polymers can reflect most directly a nature of the electron conductivity. The role of $\mu S R$ with the $\mu^{+}$is significant in this respect as explained by the following scenario.

During the slowing-down process in soft materials like conducting polymers, the injected $\mu^{+}$picks up one electron to form a neutral atomic state called muonium, which is analogous to a hydrogen atom. The muonium is then thermalized followed by chemical bonding to a reactive site on the molecule. Depending then upon the nature of the molecule, the electron brought-in by the $\mu^{+}$can show several characteristic behaviours, including localization to form a radical state and/or a linear motion along the molecular chain. These behaviours can be detected most sensitively by measuring the spin relaxation process of the $\mu^{+}$using the $\mu S R$ method; muon spin relaxation occurs through a magnetic interaction between the $\mu^{+}$and the localized and/or moving electron produced by the $\mu^{+}$itself.

This idea of the sensitive detection of the electron behaviour in macromolecules using muons has been

Table 2. The $1 / 8$ problem in high $T_{C}$ superconductors.

\begin{tabular}{|c|c|c|c|c|}
\hline Sample & & Superconductivity & Crystal structure & Magnetism \\
\hline $\mathrm{LaBa}$ & $\begin{array}{l}\mathrm{La}_{2}-\mathrm{Ba}_{x} \mathrm{CuO}_{4} \\
x=0 \cdot 125(1 / 8)\end{array}$ & Non-SC & LTT & Antiferro \\
\hline $\mathrm{La}(\mathrm{Nd}) \mathrm{Sr}$ & $\begin{array}{l}\mathrm{La}_{1.6-x} \mathrm{Nd}_{0.4} \mathrm{Sr}_{x} \mathrm{CuO}_{4} \\
x=0.12\end{array}$ & Non-SC & $\begin{array}{l}\text { LTT } \\
\text { Spin/charge } \\
\text { Stripe structure }\end{array}$ & Antiferro \\
\hline $\mathrm{LaSr}$ & $\begin{array}{l}\mathrm{La}_{2}-\mathrm{Sr}_{\mathrm{x}} \mathrm{CuO}_{4} \\
x=0.115\end{array}$ & Non-SC and SC & LTO & Antiferro \\
\hline BiSCO & $\begin{array}{l}\mathrm{Bi}_{2} \mathrm{Sr}_{2} \mathrm{Ca}_{1} \mathrm{Y}_{x} \mathrm{Cu}_{2} \mathrm{O}_{8+d} \\
c_{\text {hole }}=0.125\end{array}$ & Non-SC & & $\begin{array}{l}\text { Some spin- } \\
\text { dynamics }\end{array}$ \\
\hline
\end{tabular}


applied successfully in the studies of the electron transport in conducting polymers. A soliton-like motion has been studied for the $\mu^{+}$-produced electron in trans-polyacetylene, which contrasts with the localization seen in cispoly-acetylene following the formation of a radical state (Nagamine et al 1984; Ishida et al 1985). Similarly, polaron-type electron transport phenomena in polyaniline has also been studied (Pratt et al 1997).

The most significant observables in these $\mu$ SR studies can be summarized as follows. In longitudinal relaxation measurements, due to the nature of the interaction between the moving electrons and the stationary muons, the characteristic dimensionality of the electron motion can be studied by varying the externally applied magnetic field $\left(B_{\text {exi }}\right)$ and the following associated dependence of the muon spin relaxation rate $\left(\lambda_{\mu}\right)$; for one-dimensional electron motion $\lambda_{\mu} \propto\left(B_{\text {ext }}\right)^{-1 / 2}$, for two-dimensional electron motion $\lambda_{\mu} \propto\left(\alpha-\beta \log B_{\text {ext }}\right)$, where $\alpha$ and $\beta$ are constants, and for three-dimensional electron motion $\lambda_{\mu}$ does not usually have significant $B_{\text {ext }}$ dependence (Buttler et al 1976).

Progress has been made in the theoretical understanding of this paramagnetic relaxation process by Risch and Kehr who considered the direct stochastic treatment of the random-walk process of a spin which rapidly diffuses along a topologically one-dimensional chain (Risch et al 1992). An error-function type longitudinal relaxation function (hereafter called the R-K function), $G(t)=$ $\exp (\Gamma t) \operatorname{erfc}(\Gamma t)^{1 / 2}$, was proposed for $\lambda t_{\max } \gg 1$, where $\lambda$ is the electron spin flip rate, $t_{\max }$ the experimental time scale, and $\Gamma$ the relaxation parameter. In this theoretical treatment, in the case of topologically onedimensional electron motion, $\Gamma$ is proportional to $1 / B_{\text {exx }}$. In a recent experiment, the usefulness of $\mathrm{R}-\mathrm{K}$ function has been confirmed experimentally for the polaronic motion of conducting electrons in polyaniline (Pratt et al 1997).

\subsection{Rapid electron transfer in protein}

Electron transfer in macromolecules, such as protein, is the most important mechanism for various biological phenomena. A number of experimental investigations have been carried out to explore the electron transfer phenomena in proteins and related chemical compounds. However, almost all the existing informations on the electron transfer have been obtained by essentially macroscopic methods, which measure the evolution of the complete electron transfer from donor to acceptor.

Among various types of proteins, cytochrome $c$ attracts much attention, since it plays an essential role for example in the respiratory electron transport chain in mitochondria; it holds a position next to the final process of the cycle and transfers electrons to the surrounding oxidase complex.
In order to obtain microscopic information on electron transfer in a macromolecule, the muon spin relaxation $(\mu \mathrm{SR})$ method, as described in the latest sub-section, offers a great potential. Depending upon the nature of the molecule, the electron brought by the $\mu^{+}$into the molecule can take a characteristic behaviour including localization to form a radical state, and/or a linear motion along the molecular chain, etc. These behaviours can most sensitively be detected by measuring the spin relaxation process of the $\mu^{+}$using the $\mu S R$ method.

Experiments on the $\mu^{+}$relaxation in cytochrome $c$ have been conducted by using an intense pulsed beam ( $70 \mathrm{~ns}$ pulses at $50 \mathrm{HZ}$ repetition rate) of $4 \mathrm{MeV} \mu^{+}$at the RIKEN-RAL (Nagamine et al 1999).

At each of the measurement temperatures, the $\mu^{+}$ relaxation function, which corresponds to a timedependent change of the $\mu^{+}$polarization, was found to have an external field dependence (see figure 4 ). The observed relaxation functions $G(t)$ were fitted with the $\mathrm{R}-\mathrm{K}$ function, and the longitudinal relaxation parameter $\Gamma$, obtained at various temperatures, is seen to decrease monotonically with increasing $B_{\text {ext }}$. On closer inspection of the $B_{\text {ext }}$ dependence of $\Gamma$, there seems to be two components, (i) a weak-field dependence region (lower field), and (ii) a $\left(B_{\mathrm{ext}}\right)^{-1}$ dependence region (higher field) (figure 4). The latter region exhibits the characteristic $\mu^{+}$spin relaxation behaviour due to a linear motion of a paramagnetic electron. The critical field (hereafter, we refer to this as the cutoff field) where the second region becomes significant over the first region has a significant temperature dependence; the cutoff field is seen to reduce with decreasing temperature. It can be seen that the temperature dependence of the cutoff field can be represented by the sum of two activated components of the form $\exp \left(-E_{\mathrm{a}} / k T\right)$, where $E_{\mathrm{a}}$ is an activation energy; one with an activation energy of $150 \mathrm{meV}$ (dominant above $200 \mathrm{~K}$ ) and the other with an activation energy of less than $2 \mathrm{meV}$ (dominant below $200 \mathrm{~K}$ ). A native picture based upon the previous $\mu^{+} S R$ studies on high molecular weight conducting polymers would suggest an increase in the effective dimensionality of the diffusion rate. In the context of protein, such as cytochrome $c$ with coils and folds in its structure, the 'inter-chain' diffusion might perhaps be interpreted as 'inter-loop' jumps, which could well be strongly activated by the increased thermal displacement of the polymer occurring above the glass transition.

The most important unknown factors in the present $\mu^{+} S R$ studies are the distribution of locations of the $\mu^{+}$ bonding sites with corresponding uncertainty in the electronic structure of the $\mu^{+}$and the site from where the electron starts its linear motion. For this purpose, muon spin RF resonance as well as level crossing resonance will be most helpful. These experiments are currently in progress. 
Although, as mentioned above, there are still some unknown factors at this stage concerning the detailed nature of the probe state, it is clear that the electron transfer process through a microscopic section of cytochrome $c$ was directly detected in the present experiment.

The $\mu^{+}$SR measurement, where high efficiency of the measurements should be emphasized, can easily be extended to cytochrome $c$ in various chemical and biological environments. Most importantly, because of original high energy nature of the probe, this method can be applied to a protein in vivo. As one example for the future, it might be possible to use the technique to obtain new information on the basic functions of brain activity.

\section{Future directions}

\subsection{Very intense muon beam production}

Encouraged by the development of the design work on the front-end part of the $\mu^{ \pm}$colliders (Neuffer 1994), design of the next-century muon channel, namely superintense superconducting muon channel is now proposed.
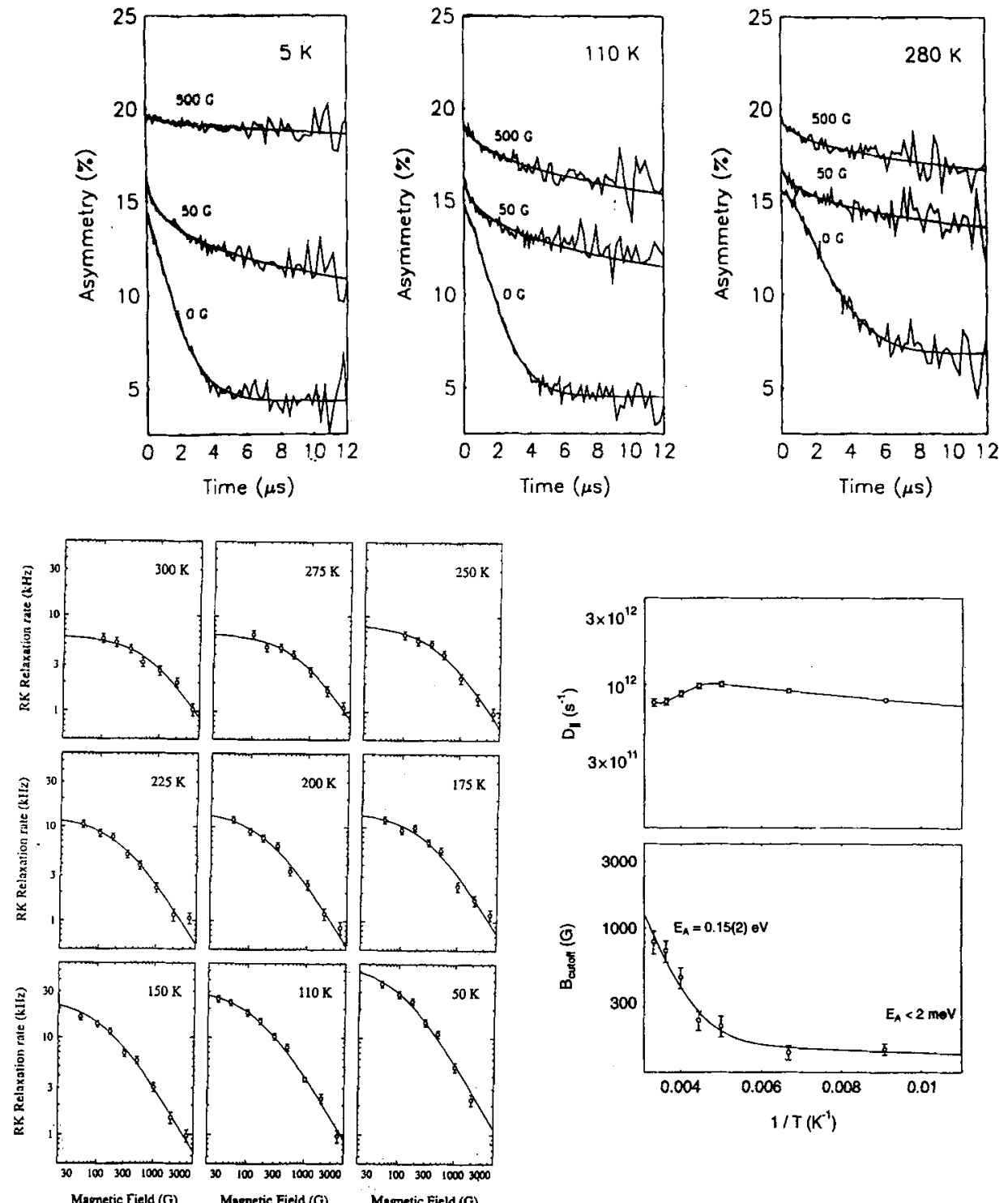

Magnetic Field (G)

Magnetic Fieid (G)

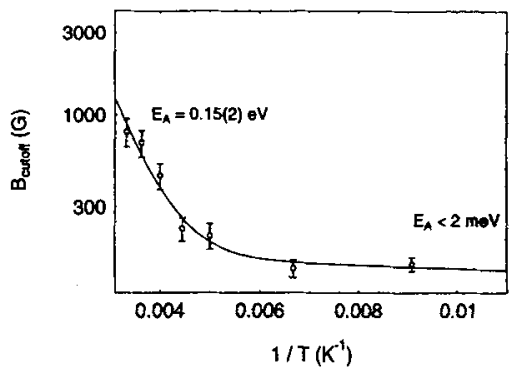

Figure 4. Typical $\mu^{+}$spin relaxation time spectra in cytochrome $c$ at $5 \mathrm{~K}, 110 \mathrm{~K}$ and $280 \mathrm{~K}$ under external longitudinal fields of $0 \mathrm{G}, 50 \mathrm{G}$ and $500 \mathrm{G}$ (above). For finite field the curves shows best fits using the $R-K$ function. The $R-K$ relaxation parameter $\Gamma$ versus external longitudinal magnetic field for the $\mu^{+}$in cytochrome $c$ at various temperatures (below left). The upper plot shows the temperature dependence of the inter-site diffusion rate of electron derived from $\mathrm{B}^{-1}$ dependent part of $\bullet$ and the lower plot shows the cutoff field against inverse temperature (below right). 
There, after placing a large-acceptance pion capture solenoid, which is similar to the front-end structure of the present design of $\mu^{ \pm}$colliders, a long decay solenoid with a RF rotation cooling capability will be installed which can be connected to the ultra-slow $\mu^{+}$production described later.

A preliminary optics design work has been completed. At RIKEN-RAL, it might be possible to install the large-acceptance pion collector at the new external proton beam line which might be constructed in relation to the Second Target Station for spallation neutron source. Some design calculations have been made. By installing a realistic pion collection solenoid and a decay solenoid with a reasonable length, one can expect a production of more than $10^{12} \mu^{+} / \mathrm{s}$.

\subsection{Ultra-slow muon beam}

Since the late $80 \mathrm{~s}$, there have been several proposals for the generation of ultra-slow $\mu^{+}$. Some of these proposals are as follows: (i) laser resonant ionization of thermal muonium $(\mathrm{Mu})$ in vacuum produced from hot metal surface (Miyake et al 1995; Nagamine et al 1995); (ii) degrading energetic $\mu^{+}$in rare gas solid (Ar, Kr, Xe) which has a finite band-gap (Morenzoni et al 1994); (iii) phase space compression of energetic $\mu^{+}$by deceleration and acceleration by electric fields (Taqqu 1986); and (iv) ionization cooling or frictional cooling by one-directional accelerating under homogenizes slowingdown (Neuffer 1994). Only the first method, however, seems to be the most relevant method for production of high-intensity and high-brightness $\mu^{+}$beam with a low emittance enough to be used for the muon science studies in the future. The earlier two methods are now being extensively applied to the $\mu^{+} S R$ spectroscopy on very thin materials and surface magnetism.

There are two processes for a realization of ultra-slow $\mu^{+}$source by the thermal-Mu-ionization method: (i) thermal $\mathrm{Mu}$ production in vacuum by stopping $\mu^{+}$at rear side of the metallic foil, followed by the $\mu^{+}$diffusion towards the foil surface, and subsequent $\mathrm{Mu}$ evaporation from the surface, and (ii) efficient muonium ionization, for example by laser resonant ionization, via $1 s \rightarrow 2 p$ $\rightarrow$ unbound excitation.

In order to realize ultra-slow $\mu^{+}$production, a new laboratory space with a dedicated pulsed $(50 \mathrm{~ms}$ width and $20 \mathrm{~Hz}$ repetition rate) proton beam line from the $500 \mathrm{MeV}$ booster synchrotron was constructed at KEKMSL. Hot tungsten (W) was adopted as the thermal Mu producing target. All of the target area as well as the following ion optics of slow $\mu^{+}$transport was maintained at a pressure below $10^{-8}$ torr under the conditions of proton-beam delivery and target heating. The actual target comprised of $2 \mathrm{~mm}$ thick boron nitride (BN) and $50 \mu \mathrm{m}$ thick W. The BN, located beside the $2300 \mathrm{~K}$ hot $\mathrm{W}$ foil, was used for efficient $\pi^{+}$production with the minimum allowable divergence of the proton beam.
For an efficient ionization method of thermal $\mathrm{Mu}$ produced by pulsed primary protons, we adopted a resonant ionization scheme using pulsed lasers namely, the single photon resonant transition of $1 s \rightarrow 2 p(122 \mathrm{~nm})$ followed by the photo-ionization transition $2 p \rightarrow$ unbound $(<366 \mathrm{~nm})$. For this purpose, a laser system was constructed for intense $122 \mathrm{~nm}$ VUV light with a $200 \mathrm{GHz}$ frequency width (FWHM), which was matched to the Doppler broadening of the thermal Mu.

During the course of the development of the ultra-slow $\mu^{+}$production, the following new experimental findings were obtained: (i) By measuring yield of the ultra-slow $\mu^{+}$as a function of temperature, the activation energy for thermal $\mathrm{Mu}$ production from hot $\mathrm{W}$ surface was obtained. Similar measurements have been carried out for the thermal $T, D$ and $H$, all of which are produced by nuclear reactions, yielding the mass-independent values of activation energies, suggesting a thermionic emission mechanism of the hydrogen-like neutrals from the hot W surface. (ii) The ultra-slow $\mu^{+}$yield, at this stage of the project development, becomes $10 \mu^{+} /[\mathrm{s} \cdot \mu \mathrm{A}$ (500 MeV proton) $10 \mu \mathrm{J} /$ pulse (112 nm laser power)]; (iii) Polarization of the produced ultra-slow $\mu^{+}$by a spin-unresolved $1 s \rightarrow 2 p \rightarrow$ unbound laser ionization was found to be $50 \%$.

By using this ultra-slow $\mu^{+}$beam, it is possible to apply the $\mu \mathrm{SR}$ method to ultra thin sample in an atomic scale thickness. Roughly speaking, the range of $10 \mathrm{keV}$ $\mu^{+}$is $50 \mathrm{~A}$, confirmed in the actual experiment conducted at KEK-MSL (Miyake et al 1997). Thus, using the $\mu$ SR method for the $\mu^{+}$stopping on the surface of magnetic materials, the following studies of the surface magnetism are possible.

(I) Spin dynamics of surface magnetism. Static order of two-dimensional magnetism exhibited in the surface magnetic materials has been studied by various experimental methods. However, dynamical behaviour, because of lack of sensitivity, has not been studied. Already by some brute-force $\mu \mathrm{SR}$ method with $2 \mathrm{MeV} \mu^{+}$applied on a stack of artificial magnetic $\mathrm{MnSb}$ superlattice, it has been pointed out that a critical slowing down has an anomalously long tail towards higher reduced temperature (Torikai et al 1994).

(II) Detection of surface reconstruction effect on magnetic properties. Recent discovery of photo-excitation response in the CMR materials indicates an importance of a surface reconstruction due to various external perturbations. Systematic studies can be done by probing lattice structure with nuclear dipolar broadening exhibiting in $\mu^{+}$spin relaxation.

\subsection{The third generation $\mu S R$ studies}

Combining ultra-slow $\mu^{+}$generation and the super-super muon channel, as seen in figure 5 , intense and extremely high emittance $\mu^{+}$beam can be realized. The expected 


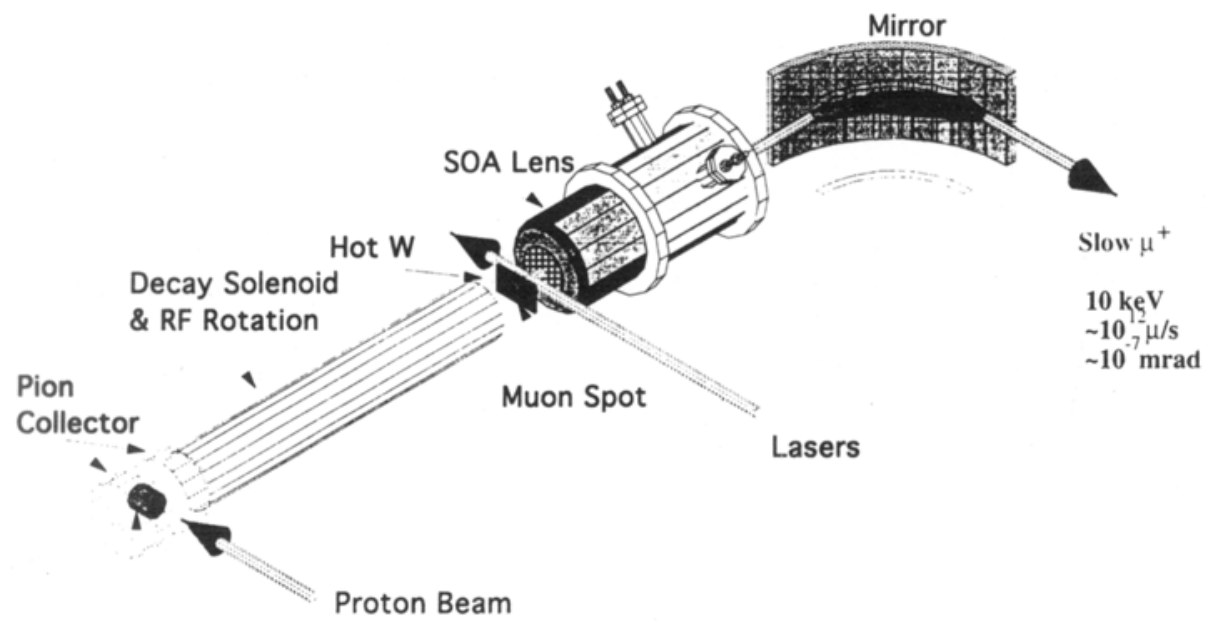

Production Target

Figure 5. Intense ultra-slow $\mu^{+}$production by the large acceptance pion collector and the decay-solenoid connected to the zero-energy cooling of the laser ionization of $\mathrm{Mu}$ from hot $\mathrm{W}$.

feature of low polarization can be overcome by adopting repolarization scheme; the thermal $\mathrm{Mu}$ can be fully repolarized by adopting spin-exchange collision between intense thermal $\mathrm{Mu}$ and optically pumped $\mathrm{Ar}$ gas. By ionization or repolarized thermal $\mathrm{Mu}$ with laser resonant ionization, intense and fully polarized ultra-slow $\mu^{+}$beam can be generated.

New generation of $\mu^{+}$beam thus obtained can realize new type of the $\mu S R$ studies. By developing focussing or slicing optics, a thin and small crystal with a volume of less than $\mu$ l can become the objective of the $\mu S R$ studies. Thus, new aspects of static and/or dynamic magnetic properties of clusters, mesoscopic systems can be explored.

On the other hand, by using $10^{1 \%} / \mathrm{s}$ level of high intensity pulsed polarized $\mu^{+}$, combined with a highly segmented $\mu$ SR spectrometer such as MWPC, one can measure the $\mu S R$ spectrum for the $\mu^{+}$stopping in a combined sequence of space and time with a unit of $\mu \mathrm{l}$ and $\mathrm{ms}$, respectively. By this space-time segmented $\mu$ SR measurement, new insights of brain function can be studied. As described in $\S 2.3$, the electron transfer imaging and its time dependent change will inform us a mechanism of consciousness for the first time.

\section{Conclusion}

As described in the present short report, the $\mu$ SR studies on magnetism and magnetic materials have marked consistent progresses and have established an unique position among various other methods of magnetism studies. At the same time muon science research, covering not only $\mu$ SR condensed matter studies but also muon $\left(\mu^{-}\right)$-catalyzed nuclear fusion, muon $\left(\mu^{-}\right)$X-ray application to non- destructive element analysis, muon $\left(\mu^{+}, \mu^{-}\right)$fundamental physics, etc has also advanced with the progress of intense particle accelerator, which in turn has motivated development of accelerator and beam technology. As mentioned in the last reaction, the $\mu \mathrm{SR}$ studies on magnetic materials will further progress in the next century.

\section{Acknowledgements}

The author would like to express his sincere thanks to the $\mu$ SR group for their collaborative efforts at both KEK-MSL and RIKEN-Muon: Drs F Pratt, V Krishnamurthy, I Watanabe, $\mathrm{H}$ Higemoto, $\mathrm{K}$ Nishiyama, $\mathrm{E}$ Torikai and many others. For providing future directions, the author would like to acknowledge contributions by Drs K Ishida, T Matsuzaki, S N Nakamura, Y Miyake and $\mathrm{K}$ Shimomura. For helpful and enlightening discussions, he would like to acknowledge many distinguished condensed-matter physicists, in particular, Professors T P Das, K Kehr, N Go and many others.

\section{References}

Amato A 1997 Rev. Med. Phys. 691119

Arai J et al 1998 Private communication

Butler M A et al 1976 J. Chem. Phys. 643592

Higemoto $W$ et al 1998 Private communication

Ishida K et al 1985 Phys. Rev. Lett. 552209

Krishnamurthy V et al 1999a Physica B259-261 374

Krishnamurthy V et al 1999b Phys. Rev. B (in press)

Kumagai K et al 1991 Physica C185-189 913

Miyake Y et al 1995 Nucl. Instrum. B95 265

Miyake Y et al 1997 Hyperfine Int. 106237

Morenzoni E et al 1994 Phys. Rev. Lett. 722793 
Nagamine K et al 1984 Phys. Rev. Lett. 531763

Nagamine K et al 1995 Phys. Rev. Lett. 744811

Nagamine K 1997 in Proceedings of the 7th international conference on $\mu S R$ (eds) K Nagamine, K Nihsiyama, R M Macrae and R Kadono (Nikko: Baltzer Sci. Publisher)

Nagamine K et al 1999 RIKEN Rev. 2051

Nishida N et al 1987 J. Appl. Phys. 26 L1856

Neuffer D V 1994 Nucl. Instrum. A350 27

Pratt F L et al 1997 Phys. Rev. Lett. 792855
Risch R èt al 1992 Phys. Rev. B46 5246

Suzaki T et al 1998 Phys. Rev. B57 57

Taqqu D 1986 Nucl. Instrum. A274 288

Torikai E et al 1993 Hyperfine Interactions 79 909, 915 , 921

Torikai E et al 1994, Hyperfine Interactions 35151

Tranquada J H et al 1995 Nature 375561

Watanabe 1 et al 1994 Hyperfine Interactions 86603

Yamamoto Y et al 1999 Phys. Rev. Lett. (submitted) 